Volume 8, No.3, May - June 2019

International Journal of Advanced Trends in Computer Science and Engineering

Available Online at http://www.warse.org/IJATCSE/static/pdf/file/ijatcse66832019.pdf

https://doi.org/10.30534/ijatcse/2019/66832019

\title{
Comparative Analysis of LTE-LAA and Wi-Fi Systems in Various Domains
}

\author{
Mandeep Singh Ramdev ${ }^{1}$, Rohit Bajaj ${ }^{2}$ \\ ${ }^{1}$ Chandigarh University, India, mandeep.singh.phd@gmail.com \\ ${ }^{2}$ Chandigarh University, India, rohit.cs@cumail.in
}

\begin{abstract}
The focus of this paper is the development of a solution regarding the coexistence of the LTE-LAA and Wi-Fi networks in the $5 \mathrm{GHz}$ bandwidth by using an interference analysis and traffic optimization for which channel selection schemes are used. By implementing an analysis tool based on the Monte Carlo method, named "3DMCAT", a simulation is applied with the LBT protocol in both the time and frequency domains on the frame based equipment of the spatial, time, spectrum domain; this tool is implemented by combining a Microsoft Excel calculation and the corresponding VBA. 3DMCAT shows the results of transmission performance analysis for the coexistence schemes of the $\mathrm{Wi}-\mathrm{Fi}$ and LTE-LAA systems in the $5 \mathrm{GHz}$ frequency bands
\end{abstract}

Keywords : Coexistence; LTE-LAA; Wi-Fi; 3DMCAT; LBT; Interference analysis

\section{INTRODUCTION}

The transmission bandwidth of the system was increased by using Carrier Aggregation (CA) between the existing licensee and the unlicensed band.

This increases the frequency-efficiency of the unlicensed band, reducing the cost of securing additional frequencies, such as License Assisted Access (LAA), which can satisfy the needs of mobile terminals. However, fair-ness issues arise between the LTE system operating exclusively in the licensed band and the WiFi system using the competition-based wireless channel access in the unlicensed band. The effect of unnecessary radiation due to the frequency separation between the adjacent WiFi AP and LTE-LAA interference is expected to occur. Therefore, a mutual coexistence technology plan needs to be established for using the frequency jointly through the analysis of the quantitative coexistence effect related to this.

The setup time of these networks is considerably less than the wired one. No need for fixed infrastructure is the prime factor that increases its applicability [9].
LTE networks comprise of several sensor nodes deployed randomly in a field having limited memory, processing capacity and power mounted on one board which cannot be recharged or replaced [10].

\section{PROPOSED SCHEME 3DMCAT ANALYSIS}

We propose the application of a Spatial, Time, Spectrum Domain (STSD), Listen Before Talk (LBT) named "3DMCAT" for a coexistence analysis regarding the Wi-Fi and LTE-LAA systems in the $5 \mathrm{GHz}$ frequency bands. Based on an interference analysis in which the SEAMCAT tool [1] was used, the time domain LBT analysis of 3DMCAT [2] involves a combination for which the Monte Carlo method is applied in the time and frequency domains. Regarding the coexistence scenario comprising the $5 \mathrm{GHz}$, Wi-Fi, and LTE-LAA systems, the 3DMCAT software process is shown in Figure 1.

\subsection{SCENARIO ANALYSIS FOR 3DMCAT}

Based on the SEAMCAT Monte Carlo principle, the random process is applied in the 3DMCAT according to trials. Each trial is one observation for one snapshot of configuration. As shown in the schematic of the overall process in Fig. 1, the 3DMCAT is implemented for the collision and interference analyses of the Wi-Fi and LTE-LAA coexistence, and is based on the Frame Based Equipment (FBE) [3] and LBT protocols [2].

\subsection{INITIALIZATION OF RANDOM POSITIONS AND TIMES}

The scenario of the Cluster/Pico cell model shown in Fig. 2 is implemented. The model is based on the inclusion of several clusters in each macro cell, whereby each cluster contains many Picos, and the maximum number of Picos depends on the minimum distance between each Pico. The minimum distance between each Pico is approximately $10 \mathrm{~m}$, and each cluster has from four to eight Picos [3]. 3DMCAT is supportive each cluster has 12 Picos and the minimum distance between each Pico is approximately $30 \mathrm{~m}$. At the beginning of the calculation, the time is generated randomly for each device (Wi-Fi or LTE) to determine the Clear Channel Assessment (CCA) start time, the transmission time, and the idle time. Because the times are randomized snapshot-by-snapshot, the calculation will sort again by ascending order for the attainment of a priority transmission decision; moreover, the location of each Pico is also randomized in the cluster's coverage range. The process must 
ensure that the distance between any pair of Picos is greater than the minimum distance value.

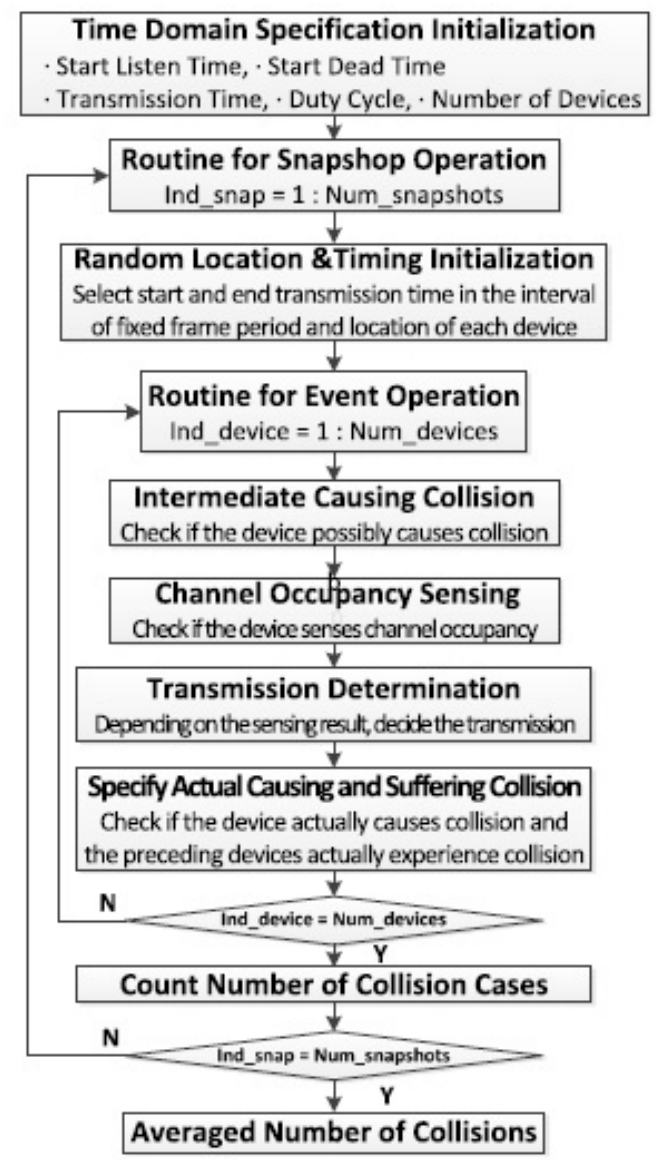

Figure 1: Overall process of 3DMCAT software.

\subsection{METHODOLOGY OF 3DMCAT}

Each snapshot of the 3DMCAT methodology is shown in Fig. 3. The number of snapshots is determined at the initializaation step. To ensure reliability, the number of snapshots must be as large as infinite (for a simulation, it is possible to accept from 5000 to 10,000 snapshots). At the beginning of the 3DMCAT, the configurations of the general time parameters are based on [2], while the FBE [3] considers the duty cycle, listen time, dead time, transmit time, duty cycle, sample time, and interval. The occupied detection is explained in Fig. 3. In Fig. 3(a), the collision that affects transmission $\# 1$ and transmission $\# 2$ is caused by a time overlapped area, whereby one transmission starts before another has ended. In Fig. 3(b), due to the absence of overlapped time, neither of the two transmissions is affected by a collision problem. The running of LBT-sensing detection is considered between the listen time and the transmit time.

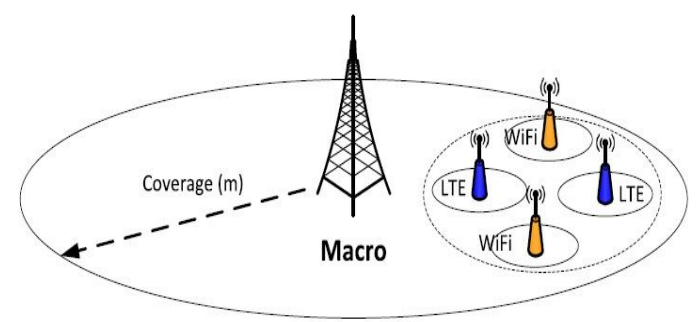

Figure 2: Cluster/Pico cell model in coexistence scenario.

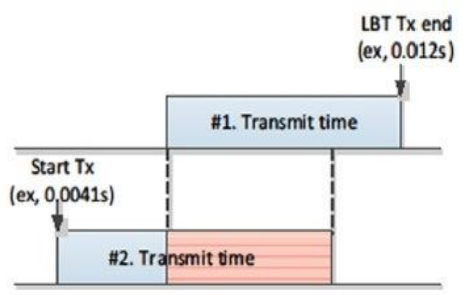

(a) True $=1$, Collision

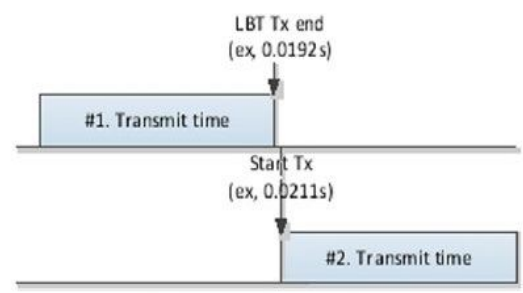

(b) False $=0$, No collision

Figure 3: Occupied detection of the 3DMCAT.

The actual collision sensing and causes of suffering are shown in Fig. 4. The collision shown in Fig. 4(a) is caused by the detection of the listen time when the previous transmission is still incomplete; otherwise, no collision occurs in Fig. 4(b).

Following the Extended Hata transmission path loss model of [4], the receiver power is calculated using the following formula:

$$
P_{R X}=P_{T X}-P_{\text {Loss }}-P_{D}
$$

where $\mathrm{P}_{\mathrm{RX}}$ is the received power, $\mathrm{P}_{\mathrm{TX}}$ is the transmitted power, and $\mathrm{P}_{\text {Loss }}$ is the transmission loss that is calculated based on the path loss equations in [4]. Also, $\mathrm{P}_{\mathrm{D}}$ is the degradation of power, whereby the adjacent channel leakage ratio (ACLR) approach, as discussed in [5] and [6], is adopted for the co-channel and the adjacent channel. For this step, a threshold is set up for interference limitation such as the interference to noise $(\mathrm{I} / \mathrm{N})$ ratio or the carrier to interference $(\mathrm{C} / \mathrm{I})$ ratio [7]; based on the established threshold, the failure or success of the transmission is determined according to the emission interference it causes for other transmissions.

With the causes of collision, the consideration scenario considers the interference from one device to other devices, whereby the transmission is marked as a failure if at least one 
interference occupation is detected, and the corresponding device is considered a halt device. In the example in Fig. 5(a), device \#1 cannot transmit data due to the failed transmission regarding device \#3. In the backward direction with the causes of suffering, one device abides interference by other transmissions and checks the transmission availability. If an unfriendly transmission (which occurs when at least one device causes an interference that exceeds the interference threshold) is detected, the transmission is temporarily discarded; therefore, in Fig. 5(b), because unfriendly interference is transmitted from device \#3 and device \#N, the transmission of device \#1 is halted.

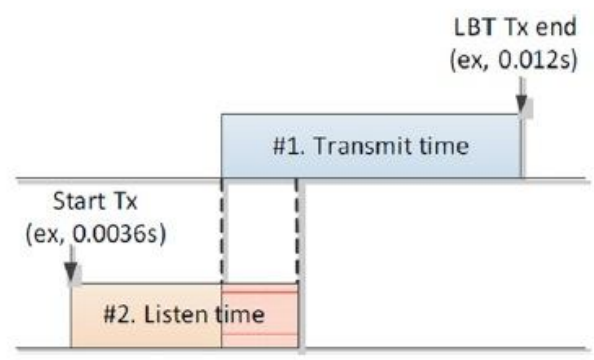

(a) True $=1$, Collision.

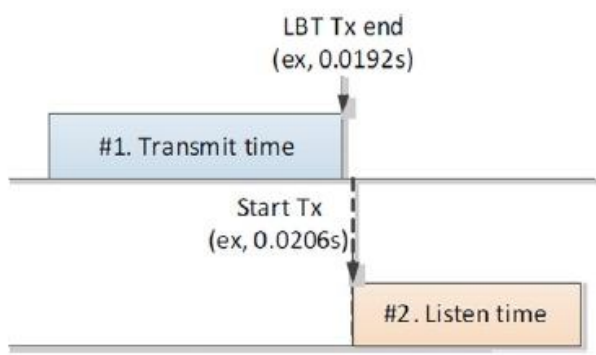

(b) False $=0$, No collision.

Figure 4: LBT sensing detection of the 3DMCAT.

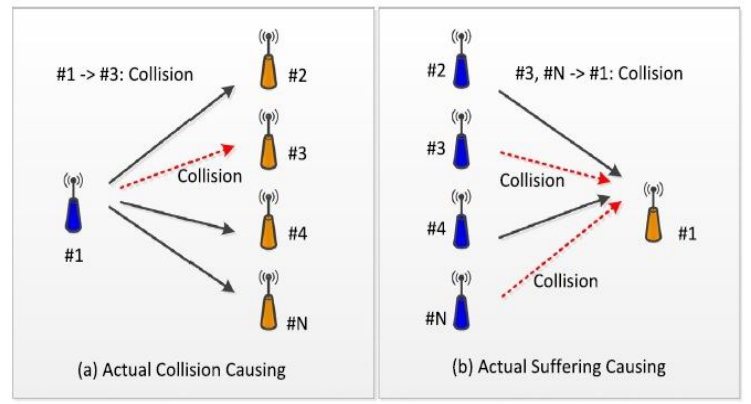

Figure 5: Actual collision and actual causes of suffering of the 3DMCAT.

Based on the Interference Calculation Engine (ICE) in [8], the successful transmission probability is defined according to the following equations. At device $\mathrm{i}^{\text {th }}$, the interference from other devices is calculated by the following equation:

iRSS $=10 \times \log \sum_{j=1}^{s} 10^{\mathrm{ins} s[j ; 1 / 10}$ where $\mathrm{S}$ is the number of Interfering Received Signal Strength (iRSS) signals and iRSS $\mathrm{S}_{[\mathrm{j}, \mathrm{i}]}$ is the iRSS from device $\mathrm{j}^{\text {th }}$ to device $\mathrm{i}^{\text {th }}$, whereby the total interference sensed at each device is the total aggregated interference from other devices, and the unit is of a $\mathrm{dBm}$ scale. The probability of a complete transmission over $\mathrm{N}$ snapshots is calculated by the following equation:

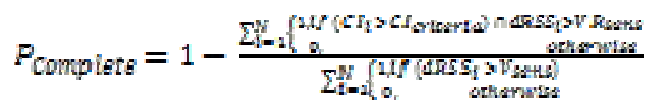

where $d R S S_{i}$ is the $d R S S$ at transceiver $\mathrm{i}^{\text {th }}, \mathrm{CI}_{\text {criteria }}$ is the CI threshold, $\mathrm{VR}_{\text {sens }}$ is the VR sensitivity, and $\mathrm{CI}_{\mathrm{i}}$ is the $\mathrm{CI}$ ratio at transceiver $\mathrm{i}^{\text {th }}$ for which the following applies:

$\mathrm{CI}_{\mathrm{i}}=d R S S_{i}-i R S S_{i}$

Here, the LBT collision detection and the actual causes of collisions are combined and run together in step 4 device-by-device until all of the devices are considered. The results are collected at the end of each snapshot until the final snapshot is reached. The general process of the transmission packet calculation is shown in Fig. 6. During this process, the incoming data packets (D) are transmitted from the transmitter to the receiver.

Table 1: Cluster/Pico-cell configuration.

\begin{tabular}{ll}
\hline Parameters & Value(s) \\
\hline Cluster coverage & $50 \mathrm{~m}$ \\
Number of Picos & 2 to 12 transceivers \\
Min. distance between two Picos & 5 to $20 \mathrm{~m}$ \\
$\Delta$ frequency & 0 to $60 \mathrm{MHz}$ \\
\hline
\end{tabular}

Table 2: FBE parameters of 3DMCAT

\begin{tabular}{ll}
\hline Parameters & Value(s) \\
\hline & \\
Listen time & $0.0002 \mathrm{~s}$ \\
Dead time & $0.00048 \mathrm{~s}$ \\
Transmit time & $0.01 \mathrm{~s}$ \\
Duty cycle & $10 \%$ \\
Sample time & $0.00002 \mathrm{~s}$ \\
Interval & $0.1 \mathrm{~s}$ \\
\hline
\end{tabular}

At the transmitter, two types of packets are presented: Incoming (D) packets and retransmit packets (R). The $\mathrm{R}$ 
packets are unsuccessful packets that were not transmitted in previous steps. Offered packets $(\mathrm{G})$ are considered for the channel occupation process. Afterward, the unsuccessful packets will be withdrawn and used as the $\mathrm{R}$ packets. The packets that were successfully transmitted $(\mathrm{T})$ are the input of the collision checking methods for the causing and suffering collisions. The collision packets (L) will be added to $\mathrm{R}$ for retransmission at the next input process. In addition, when the system is steady, the following applies:

$\mathrm{S}=\mathrm{D}$ and $\mathrm{R}=\mathrm{G}--\mathrm{S}$

Therefore, no transmissions are lost from that transmitter to the receiver, which initiates the formation of an ideal system. At the final step, three objectives will be used to determine the systemic improvement. First, LBT throughput objective shows the quality of the transmission performance based on the transmitted offered packets, as follows:

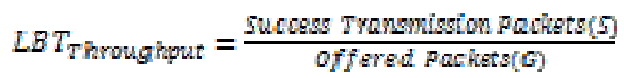

The success of the transmission packets is determined by the number of successfully received packets at the receiver side. In terms of the unsuccessful transmissions, the determining factor is the ratio of the packets lost and the retransmitted packets to the offered packets: these two fail variables represent the system halt efficiency, as follows:

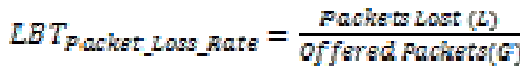

\section{IMPLEMENTATION OF 3DMCAT AND SIMULATION RESULTS}

The simulation scenario is shown in Figure 2, with the application of general LBT parameters for the Wireless LAN Access Point (WLAN AP) and LTE-LAA that are based on the FBE. The Wi-Fi and LTE-LAA transceivers use four channels in the $5 \mathrm{GHz}$ bandwidth for transmission. For the scope of this paper, the simulation will assign channels with a general priority and four channels to the current transceivers. The number of transceivers ranges from two to 12 devices, and the devices were randomly assigned to either a Wi-Fi AP transceiver or an LTE-LAA transceiver. The simulation Cluster/Pico-cell configuration is shown in Table 1. Approximately from two to 12 of the transceivers are randomly distributed in a $50 \mathrm{~m}$ radius cluster. All of the distributions must satisfy the condition in which the minimum distance between any two devices must be longer than the minimum distance of between two pico cells; the value of this minimum distance is from $5 \mathrm{~m}$ to $20 \mathrm{~m}$.

The configuration information of the 3DMCAT simulation is presented in Table 2. The Monte Carlo method comprises the input parameters for the random distribution of the positions and time generation for each of the transceivers. The calcu-lations are estimated step-by-step, snapshot-by-snapshot. The number of snapshots is defined at the beginning of the initial step, and it must be as large as possible to ensure reliability of the final results. For the experimental simulation of [1], the acceptable number of snapshots is approximately 5000 to 10,000 . The details of these parameters that are based on the FBE standardization of [3] are shown in Table 2 with the values of listen time, dead time, transmit time, duty cycle, sample time, and interval for the LBT system configuration.

The simulation in this study is conducted with two transceivers for the following three cases: Wi-Fi with Wi-Fi (Fig. 7), Wi-Fi with LTE-LAA (Fig. 8), and LTE-LAA with LTE-LAA (Fig. 9). With this simulation, a comparison is made between the Wi-Fi and LTE-LAA to determine the best performance. In addition, with a separation of the distances and the frequency difference between the two channels, the aim of the simulation is to show the throughput degradation of (6) and the PLR of (7). Based on the result for two transceivers, the throughput performance increases when a LTE-LAA and Wi-Fi coexist. Alternatively, in a scenario comprising only the LTE-LAA transceivers, a high throughput value is derived, similar to that shown in Fig. 9(a).

The minimum distance between the two transceivers and the difference between the frequencies are also controlled according to the throughput level; that is, an increase of the distance between the two transceivers causes an increase of the transmission path loss. Given the two above conditions, the interferencebetween the two transceivers will decrease; therefore, in the result, the system throughput is increased because the transmissions are more successful, and the direction of the PLR consequently decreases.

\section{CONCLUSION}

3DMCAT was applied for an analysis of the coexistence of Wi-Fi and LTE-LAA in the $5 \mathrm{GHz}$ frequency bands. Based on the process in the frequency domain comprising the Monte Carlo method and the Interference Analysis, a sound imple-mentation of 3DMCAT is shown, whereby the LBT protocol is applied in the time domain and the signal detection sensing occurs in the spatial-frequency domain. The experiment results show the performance details that facilitate the reconfiguration and analysis of a wireless system in the high frequency band-width; moreover, with a pre-defined configuration, 3DMCAT can also be applied for any wireless system in other frequency bands such as TVWS and $2.4 \mathrm{GHz}$. For future development, the proposed software will be designed with an auto channel selection function for each transceiver, and another technique will be applied to achieve results that are more objective and reliable in terms of aspects such as optimization and location probability. 
Mandeep Singh Ramdev et al., International Journal of Advanced Trends in Computer Science and Engineering, 8(3), May - June 2019, 758 - 763

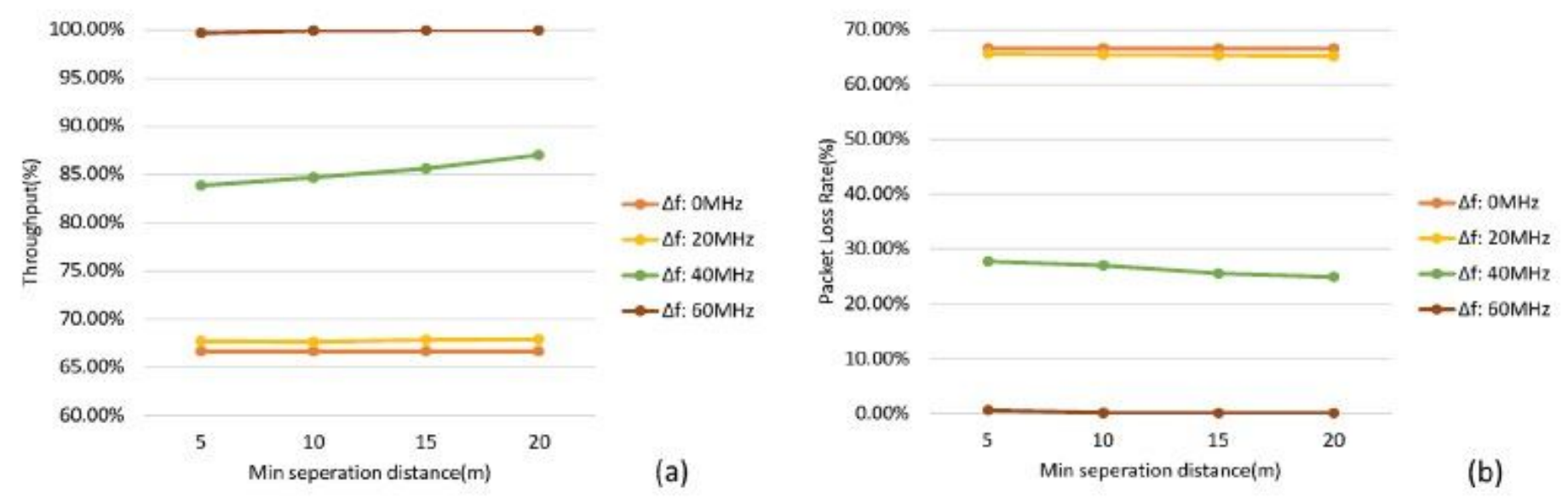

Figure 6: 3DMCAT simulation results for two transceivers: Wi-Fi and Wi-Fi.
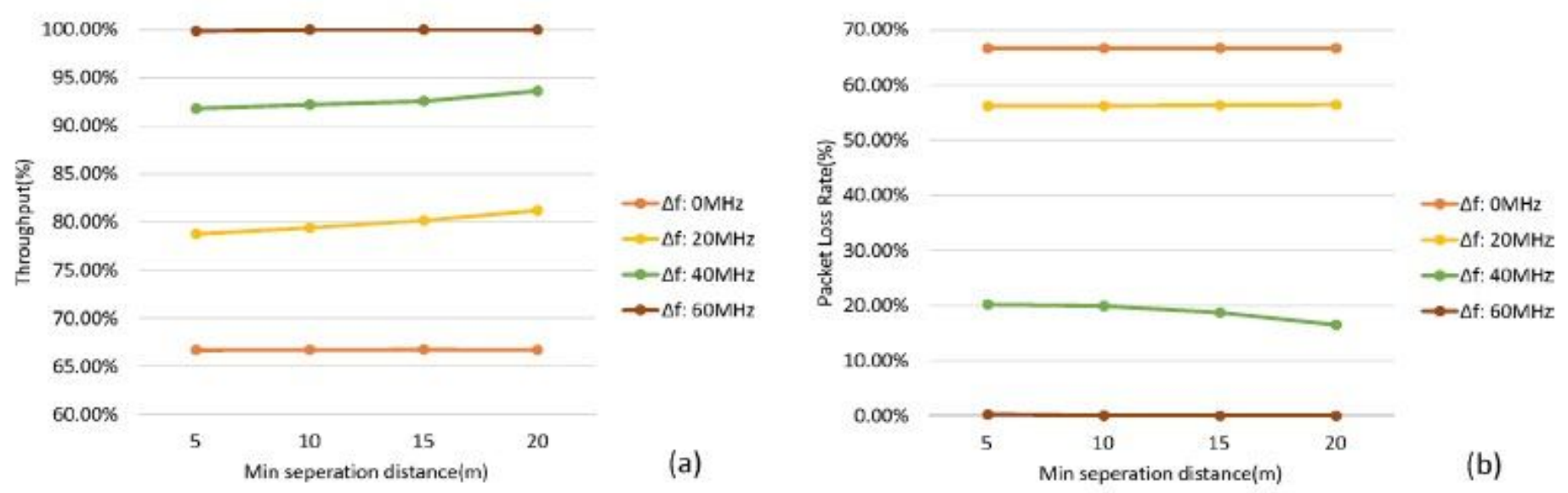

Figure 7: 3DMCAT simulation results for two transceivers: Wi-Fi and LTE-LAA.
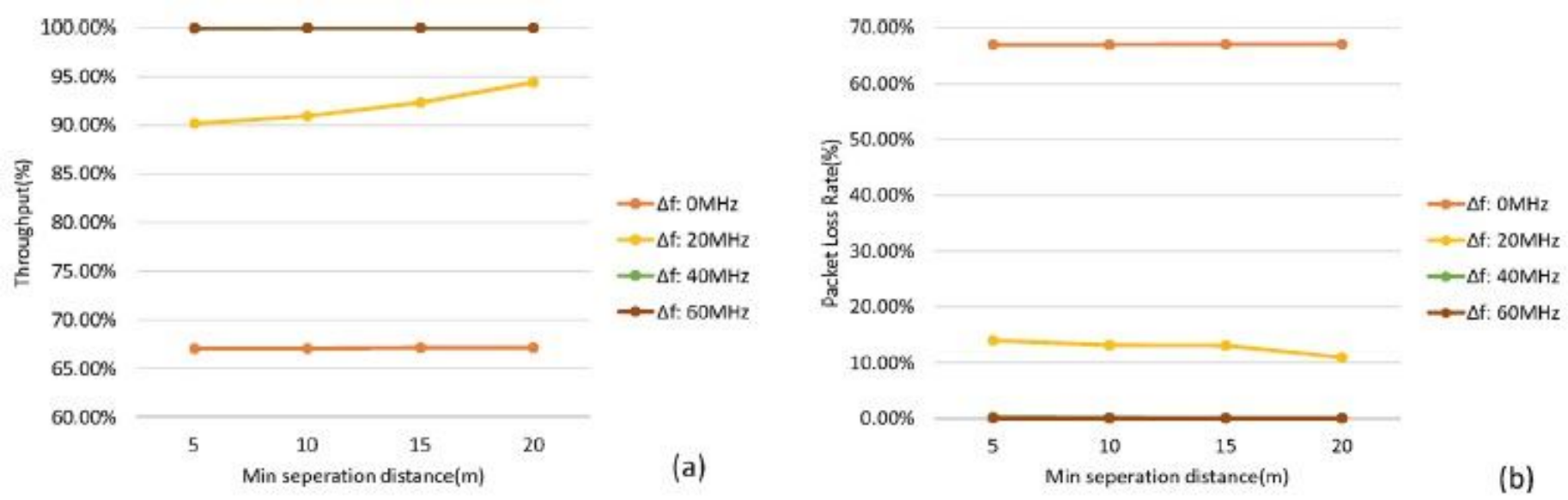

Figure 8: 3DMCAT simulation results for two transceivers: LTE-LAA and LTE-LAA. 


\section{REFERENCES}

1. SEAMCAT Handbook. Available at: http://www.seamcat.org.

2. ECC Report 181, Improving Spectrum Efficiency in the SRD Bands, ECC, Sept. 2012.

3. Qualcomm Technologies Inc. LTE-U Forum Technical Report Overview, Qualcomm, May. 2015.

4. SEAMCAT implementation of Extended hata and extended hata SRD models. Available: http://www.seamcat.org.

5. ECC Report 131, Derivation of a Block Edge Mask for Terminal Stations in the $2.6 \mathrm{GHz}$ Frequency Band, ECC, 2009.

6. Sgrignoli, G., 2003. DTV repeater emission mask analysis. IEEE transactions on broadcasting, 49(1), pp.32-80.

https://doi.org/10.1109/TBC.2003.809290

7. ECC Report 185, Complementary Report to ECC report 159 -Further Definition of Technical and Operational Requirements for the Operation of White Space Devices in the Band, pp. 470-790, ECC, Jan. 2013.

8. SEAMCET calculating the probability of interference. Available: http://w ww.seamcat.org.

9. Singh, P., Gill, N.S., "A secure and Power-Aware Protocol for Wireless Ad Hoc Networks", International Journal of Advanced Trends in Computer Science and Engineering, Vol. 8, No.1, pp 34-41

https://doi.org/10.30534/ijatcse/2019/07812019

10. Chaurasiya, H., Ghosh, S., "Performance Evaluation of Energy Efficient Cluster based Algorithms in Wireless Sensor Networks", International Journal of Advanced Trends in Computer Science and Engineering, Vol. 7, No.5, pp 77-81

https://doi.org/10.30534/ijatcse/2018/03752018 